doi:10.13108/2020-12-4-55

\title{
GEOMETRY OF RADIAL HILBERT SPACES WITH UNCONDITIONAL BASES OF REPRODUCING KERNELS
}

\author{
K.P. ISAEV, R.S. YULMUKHAMETOV
}

\begin{abstract}
We study the geometry of abstract radial functional Hilbert spaces stable with respect to dividing and possessing an unconditional basis of reproducing kernels. We obtain a simple necessary condition ensuring the existence of such bases in terms of the sequence $\left\|z^{n}\right\|, n \in \mathbb{N} \cup\{0\}$. We also obtain a sufficient condition for the norm and the Bergman function of the space to be recovered by a sequence of the norms of monomials. Two main statements we prove are as follows. Let $H$ be a radial functional Hilbert space of entire functions stable with respect to dividing and let the system of monomials $\left\{z^{n}\right\}, n \in \mathbb{N} \cup\{0\}$, be complete in this space.
\end{abstract}

1. If the space $H$ possesses an unconditional basis of reproducing kernels, then

$$
\left\|z^{n}\right\| \asymp e^{u(n)}, \quad n \in \mathbb{N} \cup\{0\},
$$

where the sequence $u(n)$ is convex, that is

$$
u(n+1)+u(n-1)-2 u(n) \geqslant 0, \quad n \in \mathbb{N} .
$$

2. Let $u_{n, k}=u(n)-u(k)-(u(n)-u(n-1))(n-k)$. If $\mathcal{U}$ is the matrix with entries $e^{2 u_{n, k}}, n, k \in \mathbb{N} \cup\{0\}$, and

$$
\|\mathcal{U}\|:=\sup _{n}\left(\sum_{k} e^{2 u_{n, k}}\right)^{\frac{1}{2}}<\infty
$$

then

2.1. the space $H$ as a Banach space is isomorphic to the space of entire functions with the norm

$$
\|F\|^{2}=\frac{1}{2 \pi} \int_{0}^{\infty} \int_{0}^{2 \pi}\left|F\left(r e^{i \varphi}\right)\right|^{2} e^{-2 \widetilde{u}(\ln r)} d \varphi d \widetilde{u}_{+}^{\prime}(\ln r),
$$

where $\widetilde{u}$ is the Young conjugate of the piecewise-linear function $u(t)$;

2.2. the Bergman function of the space $H$ satisfies the condition

$$
K(z) \asymp e^{2 \widetilde{u}(\ln |z|)}, \quad z \in \mathbb{C} .
$$

Keywords: Hilbert spaces, entire functions, unconditional bases, reproducing kernels

Mathematics Subject Classification: 46E22, 30D10

K.P. Isaev, R.S. Yulmukhametov, Geometry of radial Hilbert spaces with unconditional BASES OF REPRODUCING KERNELS.

(C)Isaev K.P., Yulmukhametov R.S. 2020.

The research of the first author is made in the framework of the development program of Scientific and Educational Mathematical Center of Privolzhsky Federal District, additional agreement no. 075-02-2020-1421/1 to agreement no. 075-02-2020-1421. The second author is supported by Russian Foundation for Basic Researches (project no. 18-01-00095-a).

Submitted September 17, 2020. 


\section{INTRODUCTION}

Throughout the paper the symbol stands $H$ for a functional Hilbert space of entire functions stable with respect to dividing in the following sense:

1) all evaluation functionals $\delta_{z}: f \rightarrow f(z)$ are continuous;

2) if $F \in H, F\left(z_{0}\right)=0$, then $F(z)\left(z-z_{0}\right)^{-1} \in H$.

The functional property of the space implies that it admits a reproducing kernel $k(\lambda, z)$ :

$$
f(z)=(f(\lambda), k(\lambda, z)), \quad \forall z \in \mathbb{C}, \quad \forall f \in H .
$$

By $K(z)$ we denote $k(z, z)$. Then the Bergman function of the space $H$ is $\left\|\delta_{z}\right\|_{H}=(K(z))^{\frac{1}{2}}$, see [1].

A basis $\left\{e_{k}, k=1,2, \ldots\right\}$ in a Hilbert space is an unconditional basis, see [2], if there exist numbers $c, C>0$ such that for each element $x=\sum_{k=1}^{\infty} x_{k} e_{k} \in H$, the identity holds:

$$
c \sum_{j=1}^{\infty}\left|c_{k}\right|^{2}\left\|e_{k}\right\|^{2} \leqslant\left\|\sum_{j=1}^{\infty} c_{k} e_{k}\right\|^{2} \leqslant C \sum_{j=1}^{\infty}\left|c_{k}\right|^{2}\left\|e_{k}\right\|^{2} .
$$

The issue on existence and construction of unconditional bases from the values of reproducing kernels in the Hilbert spaces of analytic functions is actively studied during last years.

This problems goes back to closely related classical problems: representation of the functions by exponential series and interpolation by entire functions. The representation by exponential series was actively developed by A.F. Leontiev and by its pupils, the main results and analytic methods were exposed in monograph [3]. Yu.F. Korobeinik and its pupils studied functional analytic methods, he created the theory of absolutely representing systems in locally convex spaces of holomorphic functions; the main results were presented in work [4]. In the theory of absolutely representing systems, the fineness of the topology in the space naturally plays an important role. In works [5], 6] there proved theorems on existence of representing exponential systems in projective and inductive limits of weighted spaces, in which the differentiation operator acts continuously.

The further advantage in this space in the sense of the topology fineness suggests the studying of normed spaces, that is, the construction of unconditional basis. As it turned out, the bases of exponentials is a rare event. To the best of the authors' knowledge, these are bases in the classical space $L_{2}$ and the Sobolev spaces $L_{2}^{s}$, see [7], the bases in Smirnov spaces [8] and Bergman spaces [9] on convex polygons. There is a series of works on the absence of the bases of exponentials. For instance, there are no bases of exponentials in the Smirnov and Bergman spaces on domains with a smooth boundary, see [10], [11]. There are also no bases of exponentials in the weighted spaces, when the weight grows faster than a power function [12] or is comparable with a power function [13.

In works [14]-[16], in terms of the interpolation by entire functions there was shown the absence of unconditional bases formed by the values of reproducing kernel in the classical Bargmann space and Fock spaces

$$
\mathcal{F}_{\varphi}=\left\{f \in H(\mathbb{C}):\|f\|^{2}:=\int_{\mathbb{C}}|f(\lambda)|^{2} e^{-2 \varphi(\lambda)} d m(\lambda)<\infty\right\},
$$

with radial weights $\varphi$ growing faster than $|\lambda|^{2}$. In work [17] there was proved the absence of unconditional bases formed by values of reproducing kernel in spaces with weights obeying the conditions $\left(\ln _{+} r\right)^{2}=o(\varphi(r)), r \rightarrow \infty$ and possessing some regularity of the growth. In the same work, an unexpected result was obtained, which stated the existence of unconditional bases formed by the values of a reproducing kernel in the Fock spaces $\mathcal{F}_{\varphi_{\alpha}}$ with the weights 
$\varphi_{\alpha}(\lambda)=\left(\ln _{+}|\lambda|\right)^{\alpha}$ as $\alpha \in(1 ; 2]$. Later, in paper [18, there was proved the existence of unconditional bases formed by values of a reproducing kernel in the Fock spaces with radial weights of essentially more general form.

In what follows we shall make use of the following notations. For positive functions $A, B$, the writing $A(x) \asymp B(x), x \in X$, means that for some constants $C, c>0$ and for all $x \in X$ the estimates $c B(x) \leqslant A(x) \leqslant C B(x)$ hold. The symbol $A(x) \prec B(x), x \in X,(A(x) \succ B(x)$, $x \in X)$, means the existence of a constant $C>0$ such that $A(x) \leqslant C B(x)(B(x) \leqslant C A(x))$.

A functional Hilbert space $H$ is called radial if for some $F \in H$ and $\varphi \in \mathbb{R}$, the function $F\left(z e^{i \varphi}\right)$ belongs to $H$, and

$$
\left\|F\left(z e^{i \varphi}\right)\right\|=\|F\| .
$$

It is obvious that in a radial Hilbert space the identity holds: $K\left(z e^{i \varphi}\right) \equiv K(z), z \in \mathbb{C}, \varphi \in \mathbb{R}$.

In the present work we consider abstract radial functional Hilbert spaces stable with respect to dividing and we prove the following statements.

1. If $H$ is a radial functional Hilbert space stable with respect to dividing and admitting an unconditional bases formed by values of the reproducing kernel, then there exists a convex sequence $u(n), n \in \mathbb{N} \cup\{0\}$, such that $\|z\|^{n} \asymp e^{u(n)}, n \in \mathbb{N} \cup\{0\}$. The convexity of $\{u(n)\}$ means

see Theorem 1 .

$$
u(n+1)+u(n-1)-2 u(n) \geqslant 0, \quad n \in \mathbb{N},
$$

2. Let $u_{n, k}=u(n)-u(k)-(u(n)-u(n-1))(n-k)$. If $\mathcal{U}$ is a matrix with entries $e^{2 u_{n, k}}$, $n, k \in \mathbb{N} \cup\{0\}$, and

$$
\|\mathcal{U}\|:=\sup _{n}\left(\sum_{k} e^{2 u_{n, k}}\right)^{\frac{1}{2}}<\infty
$$

then

2.1. the space $H$ as a Banach space is isomorphic to the space of entire functions with the norm

$$
\|F\|^{2}=\frac{1}{2 \pi} \int_{0}^{\infty} \int_{0}^{2 \pi}\left|F\left(r e^{i \varphi}\right)\right|^{2} e^{-2 \widetilde{u}(\ln r)} d \varphi d \widetilde{u}_{+}^{\prime}(\ln r),
$$

where $\widetilde{u}$ is the Young conjugate of a piecewise linear function $u(t)$;

2.2. the Bergman function of the space $H$ satisfies the condition

$$
K(z) \asymp e^{2 \widetilde{u}(\ln |z|)}, \quad z \in \mathbb{C} .
$$

\section{Description of norm And Bergman FunCtion}

The following theorem was proved in work [19], see Theorem 1.

Theorem 1. If in a radial functional Hilbert space $H$ stable with respect to dividing and containing all monomials $z^{n}, n=0,1,2, \ldots$, then there exists an unconditional basis formed by the values of reproducing kernel, then there exists a convex sequence $u(n), n \in \mathbb{N} \cup\{0\}$, such that $\|z\|^{n} \asymp e^{u(n)}, n \in \mathbb{N} \cup\{0\}$. At that,

$$
\lim _{n \rightarrow+\infty}(u(n+1)-u(n))=\lim _{n \rightarrow+\infty} \frac{u(n)}{n}=+\infty .
$$

If in a radial Hilbert space, the system of monomials $z^{n}, n \in \mathbb{N} \cup\{0\}$, is complete, then the space is completely determined by the sequence $u(n)=\ln \left\|z^{n}\right\|, n=0,1,2, \ldots$ This is implied by the following statement.

Lemma 1. Let $H$ be a radial Hilbert space of entire functions and $z^{n}, z^{m} \in H, n \neq m$. Then

$$
\left(z^{n}, z^{m}\right)=0 .
$$


Proof. The radial property of the space implies that for each $\varphi \in \mathbb{R}$

$$
\left\|z^{n}+z^{m}\right\|^{2}=\left\|\left(z e^{i \varphi}\right)^{n}+\left(z e^{i \varphi}\right)^{m}\right\|^{2}=\left\|z^{n}\right\|^{2}+2 \operatorname{Re} e^{i(n-m) \varphi}\left(z^{n}, z^{m}\right)+\left\|z^{m}\right\|^{2},
$$

that is,

$$
\operatorname{Re} e^{i(n-m) \varphi}\left(z^{n}, z^{m}\right) \equiv \text { Const, }
$$

which is possible only under the orthogonality of $z^{n}$ and $z^{m}$. The proof is complete.

The space of entire functions with the norm

$$
\|F\|^{2}=\sum_{n=0}^{\infty}\left|\frac{F^{(n)}(0)}{n !}\right|^{2} e^{2 u(n)}
$$

is a radial Hilbert space, in which system of monomials $z^{n}, n=0,1, \ldots$, is orthogonal and complete. Hence, in the both spaces this system forms an orthogonal basis and these spaces coincide as Banach ones.

Theorem 2. If in a radial functional Hilbert space $H$ stable with respect to dividing, the polynomials are complete and for some convex on $\mathbb{R}$ function $u(x)$ we have

$$
\begin{aligned}
& \left\|z^{n}\right\| \asymp e^{u(n)}, \quad n=0,1, \ldots, \\
& U(x)=\int_{0}^{\infty} e^{2 x t-2 u(t)} d t, \quad x \in \mathbb{R}, \\
& \widetilde{u}(x)=\sup _{t \in \mathbb{R}}(x t-u(t)), \quad x \in \mathbb{R},
\end{aligned}
$$

then the space $H$ is a Banach space is isomorphic to space

$$
\left\{F \in H(\mathbb{C}): \frac{1}{2 \pi} \int_{0}^{\infty} \int_{0}^{2 \pi} \frac{\left|F\left(r e^{i \varphi}\right)\right|^{2} d \varphi d \widetilde{u}_{+}^{\prime}(\ln r)}{U(\ln r)}<\infty\right\} .
$$

Proof. In work [20], there was introduced a characteristics of convex functions

$$
\rho_{\widetilde{u}}(x)=\sup \left\{t>0: \int_{x-t}^{x+t}\left|\widetilde{u}_{+}^{\prime}(\tau)-\widetilde{u}_{+}^{\prime}(t)\right| d \tau \leqslant 1\right\},
$$

and it was proved (in Lemma 2) that

$$
e^{-2} e^{2 u(t)} \leqslant \int_{-\infty}^{\infty} e^{2 x t-2 \widetilde{u}(x)} \rho_{\widetilde{u}}(x) d \widetilde{u}_{+}^{\prime}(x) \leqslant \frac{\pi}{2} e^{2} e^{2 u(t)}, \quad t \in \mathbb{R} .
$$

By [21, Thm. 2]

$$
U(x) \asymp \frac{1}{\rho_{\widetilde{u}}(x)} e^{2 \widetilde{u}(x)}, \quad x \in \mathbb{R},
$$

thus,

$$
\int_{-\infty}^{\infty} \frac{e^{2 x t}}{U(x)} d \widetilde{u}_{+}^{\prime}(x) \asymp \int_{-\infty}^{\infty} e^{2 x t-2 \widetilde{u}(x)} \rho_{\widetilde{u}}(x) d \widetilde{u}_{+}^{\prime}(x) \asymp e^{2 u(t)}, \quad t \in \mathbb{R} .
$$

For $F \in H$, we let

$$
\|F\|_{0}^{2}:=\frac{1}{2 \pi} \int_{0}^{\infty} \int_{0}^{2 \pi} \frac{\left|F\left(r e^{i \varphi}\right)\right|^{2} d \varphi d \widetilde{u}_{+}^{\prime}(\ln r)}{U(\ln r)}
$$


Then by (1)

$$
\left\|z^{n}\right\|_{0}^{2}=\int_{0}^{\infty} \frac{|r|^{2 n} d \widetilde{u}_{+}^{\prime}(\ln r)}{U(\ln r)}=\int_{-\infty}^{\infty} \frac{e^{2 n x} d \widetilde{u}_{+}^{\prime}(x)}{U(x)} \asymp e^{2 u(n)} \asymp\left\|z^{n}\right\|^{2}, \quad n \in \mathbb{N} \cup\{0\} .
$$

Since the system of monomials forms an orthogonal basis, these norms are equivalent. The proof is complete.

Without loss of generality we can suppose that the sequence $\ln \left\|z^{n}\right\|, n=0,1, \ldots$, is increasing and convex and $\ln \left\|z^{0}\right\|=0$.

Lemma 2. Let $u(t), u(n)=\ln \left\|z^{n}\right\|, n=0,1,2, \ldots$, be a piece-wise linear non-decreasing functions with jumps at integer non-negative points, that is,

$$
u(t)=u(n)+(u(n+1)-u(n))(t-n), \quad t \in[n ; n+1], \quad n \in \mathbb{N} \cup\{0\},
$$

and

$$
u_{+}^{\prime}(n)=u_{-}^{\prime}(n+1)=u(n+1)-u(n), \quad n \in \mathbb{N} \cup\{0\} .
$$

Then the Young conjugate $\widetilde{u}(x)$ is also piece-wise linear with jumps at the points $x_{n}=u(n)-$ $u(n-1)=u_{+}^{\prime}(n-1), n \in \mathbb{N}$.

The derivative $\widetilde{u}_{+}^{\prime}(x)$ is the function with unite jumps at the points $x_{n}, n \in \mathbb{N}$, in particular,

$$
\widetilde{u}\left(x_{n}\right)=x_{n} n-u(n), \quad \widetilde{u}_{+}^{\prime}\left(x_{n}\right)=n, \quad n \in \mathbb{N} .
$$

Proof. Since

$$
\begin{aligned}
\widetilde{u}(x) & =\sup _{t \geqslant 0}(x t-u(t))=\sup _{n \in \mathbb{N} \cup\{0\}} \sup _{\tau \in[0 ; 1]}(x(n+\tau)-(u(n)+(u(n+1)-u(n)) \tau) \\
& =\sup _{n \in \mathbb{N} \cup\{0\}}\left(x n-u(n)+\sup _{\tau \in[0 ; 1]}(x-(u(n+1)-u(n)) \tau)\right)
\end{aligned}
$$

and the internal supremum is attained at the end-points of the interval $[0 ; 1]$, then

$$
\widetilde{u}(x)=\sup _{n \in \mathbb{N} \cup\{0\}}(x n-u(n)), \quad x \in \mathbb{R} .
$$

Thus, the Young conjugate $\widetilde{u}(x)$, as an upper envelope of a sequence of linear functions, is also linear with jumps at the points $x_{n}=u(n)-u(n-1)=u_{+}^{\prime}(n-1), n \in \mathbb{N}$, or, in more details,

$$
\widetilde{u}(x)= \begin{cases}0, & x \leqslant x_{1}=u(1)-u(0), \\ 1 \cdot x-u(1), & x_{1} \leqslant x \leqslant x_{2}=u(2)-u(1), \\ \cdots & \\ n x-u(n), & x_{n} \leqslant x \leqslant x_{n+1}=u(n+1)-u(n), \\ \ldots & \end{cases}
$$

The proof is complete.

By Lemma 2, the function $\widetilde{u}_{+}^{\prime}(\ln r)$ has unit jumps at the points $R_{n}=e^{x_{n}}$ and the norm $\|F\|_{0}$ becomes the sum of a series:

$$
\|F\|_{0}^{2}=\sum_{n=1}^{\infty} \frac{1}{U\left(\ln R_{n}\right)}\left(\frac{1}{2 \pi} \int_{0}^{2 \pi}\left|F\left(R_{n} e^{i \varphi}\right)\right|^{2} d \varphi\right) .
$$

Theorem 3. Let $K(\lambda)$ be the Bergman function of a radial functional Hilbert space $H$ stable with respect to dividing, in which the system of monomials is complete. If the numbers

$$
u_{n, k}=u(n)-u(k)-u_{+}^{\prime}(n-1)(n-k), \quad n, k \in \mathbb{N} \cup\{0\},
$$


satisfy the condition

$$
\sup _{n} \sum_{k} e^{2 u_{n, k}}:=A<\infty
$$

then

$$
K(\lambda) \asymp e^{2 \widetilde{u}(\ln |\lambda|)}, \quad \lambda \in \mathbb{C} .
$$

Proof. Under the assumptions of the theorem, the functions $\left\{\frac{z^{n}}{\left\|z^{n}\right\|}, n=0,1, \ldots\right\}$ form an orthonormalized basis. Therefore,

$$
k(\lambda, z)=\sum_{k=0}^{\infty} \frac{\lambda^{k} \bar{z}^{k}}{\left\|z^{k}\right\|^{2}}, \quad K(z)=\sum_{k=0}^{\infty} \frac{\left|z^{k}\right|^{2}}{\left\|z^{k}\right\|^{2}}, \quad z \in \mathbb{C} .
$$

Let us prove the needed relation on critical circumferences $|\lambda|=R_{n}$,

$$
1 \leqslant K\left(R_{n}\right) R_{n}^{-2 n}\left\|z^{n}\right\|^{2} \asymp \sum_{k=0}^{\infty} R_{n}^{2(k-n)} \exp (-2 u(k)+2 u(n)), \quad n \in \mathbb{N} .
$$

Since $\ln R_{n}=x_{n}=u_{+}^{\prime}(n-1)$, we have

$$
R_{n}^{2(k-n)} \exp (-2 u(k)+2 u(n))=\exp \left(2\left(u(n)-u(k)-u_{+}^{\prime}(n-1)(n-k)\right)\right)=e^{2 u_{n, k}},
$$

and by the assumptions of the theorem,

$$
K\left(R_{n}\right) \asymp \frac{R_{n}^{2 n}}{\left\|z^{n}\right\|^{2}}, \quad n \in \mathbb{N} .
$$

By formula (3) we get

$$
K\left(R_{n}\right) \asymp \exp \left(2 \widetilde{u}\left(\ln R_{n}\right)\right) .
$$

The function $\ln K\left(e^{x}\right)$ is convex and by the above proved facts,

$$
\ln K\left(e^{x_{n}}\right) \leqslant \text { Const }+2 \widetilde{u}\left(x_{n}\right), \quad n \in \mathbb{N} .
$$

Since the function $\widetilde{u}(t)$ is linear between the points $x_{n}$, this relation holds for all $x$ :

$$
K(\lambda) \prec \exp (2 \widetilde{u}(\ln |\lambda|)), \quad \lambda \in \mathbb{C} .
$$

On the other hand, by the definition of the Bergman function and by formula (2), we find that

$$
\begin{aligned}
K(\lambda) & =\sup _{F \in H} \frac{|F(\lambda)|^{2}}{\|F\|^{2}} \geqslant \sup _{n \in \mathbb{N} \cup\{0\}} \frac{\left|\lambda^{n}\right|^{2}}{\left\|\lambda^{n}\right\|^{2}} \\
& =\exp \left(2 \sup _{n \in \mathbb{N} \cup\{0\}}(n \ln |\lambda|-u(n))\right)=\exp (2 \widetilde{u}(\ln |\lambda|)) .
\end{aligned}
$$

Together with (6) this implies the statement of the theorem. The proof is complete.

Corollary 1. Let a sequence $u(n)$ satisfy a condition:

$$
\inf _{n}\left(u_{+}^{\prime}(n+p)-u_{+}^{\prime}(n)\right):=\sigma>0
$$

for some $p \in \mathbb{N}$. Then

$$
K(\lambda) \asymp e^{2 \widetilde{u}(\ln |\lambda|)}, \quad \lambda \in \mathbb{C} .
$$

By Theorem 3, to prove this corollary, it is sufficient to prove the following lemma.

Lemma 3. Condition (7) implies condition (5). 
Proof. We take $n \in \mathbb{N} \cup\{0\}$ and let $k>n$. For a piece-wise linear function

$$
u_{+}^{\prime}(n)=u(n+1)-u(n),
$$

we have

$$
u(k)-u(n)=\sum_{j=0}^{k-n-1}(u(n+j+1)-u(n+j))=\sum_{j=0}^{k-n-1} u_{+}^{\prime}(n+j) .
$$

By condition $\left(u^{\prime}\right)$,

$$
u_{+}^{\prime}(n+j) \geqslant u_{+}^{\prime}(n-1)+\left[\frac{j}{p}\right] \sigma, \quad n=1,2, \ldots, \quad j=0,1, \ldots,
$$

where $[x]$ denotes the integer part of $x$. Hence,

$$
\begin{aligned}
u(k)-u(n) & \geqslant(k-n) u_{+}^{\prime}(n-1)+\sigma \sum_{j=0}^{k-n-1}\left[\frac{j}{p}\right] \\
& \geqslant(k-n) u_{+}^{\prime}(n-1)+\frac{\sigma p}{2}\left(\left[\frac{k-n-1}{p}\right]-1\right)\left[\frac{k-n-1}{p}\right],
\end{aligned}
$$

and therefore,

$$
u(n)-u(k)-u_{+}^{\prime}(n-1)(n-k) \leqslant-\frac{\sigma p}{2}\left(\left[\frac{k-n-1}{p}\right]-1\right)\left[\frac{k-n-1}{p}\right], \quad k \geqslant n .
$$

Thus,

$$
\sum_{k=n}^{\infty} e^{2 u_{n, k}} \leqslant \sum_{j=0}^{\infty} \exp \left(-\sigma p\left(\left[\frac{j}{p}\right]-1\right)\left[\frac{j}{p}\right]\right):=C(\sigma, p), \quad n \in \mathbb{N} .
$$

Let $k<n$, then

$$
\begin{aligned}
u(n)-u(k)-u_{+}^{\prime}(n-1)(n-k) & =\sum_{j=1}^{n-k}\left(u(n-j+1)-u(n-j)-u_{+}^{\prime}(n-1)\right) \\
& =\sum_{j=1}^{n-k}\left(u_{+}^{\prime}(n-j)-u_{+}^{\prime}(n-1)\right) .
\end{aligned}
$$

Since $j=s p+1, \ldots,(s+1) p, s=0,1,2, \ldots$, the inequality holds

$$
u_{+}^{\prime}(n-j)-u_{+}^{\prime}(n-1) \leqslant-s \sigma,
$$

then

$$
u(n)-u(k)-u_{+}^{\prime}(n-1)(n-k) \leqslant-\frac{p \sigma}{2}\left[\frac{n-k}{p}\right]\left(\left[\frac{n-k}{p}\right]-1\right), \quad k<n .
$$

Hence,

$$
\sum_{k=0}^{n-1} e^{2 u_{n, k}} \leqslant \sum_{j=0}^{\infty} \exp \left(-p \sigma\left[\frac{j}{p}\right]\left(\left[\frac{j}{p}\right]-1\right)\right)=C(\sigma, p), \quad n \in \mathbb{N} .
$$

By (8) this implies condition (5). The proof is complete.

Theorem 4. Let $H$ be a radial functional Hilbert space stable with respect to dividing, in which the system of monomials is complete. If condition (5) holds, then the norm of the space $H$ is equivalent to the norm

$$
\|F\|_{0}^{2}:=\frac{1}{2 \pi} \sum_{n=1}^{\infty} e^{-2 \widetilde{u}\left(\ln R_{n}\right)} \int_{0}^{2 \pi}\left|F\left(R_{n} e^{i \varphi}\right)\right|^{2} d \varphi .
$$


Proof. By Theorem 1 and formula (4), it is sufficient to show that under condition (5) we have

$$
U\left(x_{n}\right) \asymp e^{2 \widetilde{u}\left(x_{n}\right)}, \quad n \in \mathbb{N} .
$$

The lower bound is always satisfied. Indeed, by the linear property of the function $u(t)$ in the intervals $[k, k+1], k \in \mathbb{N} \cup\{0\}$, the identities

$$
U\left(x_{n}\right)=\int_{0}^{\infty} e^{2\left(x_{n} t-u(t)\right)} d t \geqslant \int_{n-1}^{n} e^{2\left(x_{n} t-\left(u(n-1)+u_{+}^{\prime}(n-1)(t-n+1)\right)\right)} d t, \quad n \in \mathbb{N},
$$

hold and since by Proposition 2,

$$
x_{n}=u_{+}^{\prime}(n-1)
$$

then in view of formula $(3)$ we have

$$
U\left(x_{n}\right) \geqslant e^{2\left(x_{n}(n-1)-u(n-1)\right)}=e^{2 \widetilde{u}\left(x_{n}\right)} .
$$

We are going to prove the upper bound on the base of Theorem 3 . The function $x_{n} t-u(t)$ is concave with respect to the variable $t$, attains its maximum at a point $t=n$. Hence, in the interval $(n ; \infty)$ the function does not increases and this is why

$$
\int_{n}^{\infty} e^{2\left(x_{n} t-u(t)\right)} d t=\sum_{k=n}^{\infty} \int_{k}^{k+1} e^{2\left(x_{n} t-u(t)\right)} d t \leqslant \sum_{k=n}^{\infty} e^{2\left(x_{n} k-u(k)\right)}=e^{2 \widetilde{u}\left(x_{n}\right)} \sum_{k=n}^{\infty} e^{2 u_{n, k}} .
$$

In the interval $(0 ; n)$ this function does not decrease and this is why

$$
\int_{0}^{n} e^{2\left(x_{n} t-u(t)\right)} d t=\sum_{k=0}^{n-1} \int_{k}^{k+1} e^{2\left(x_{n} t-u(t)\right)} d t \leqslant \sum_{k=1}^{n} e^{2\left(x_{n} k-u(k)\right)}=e^{2 \widetilde{u}\left(x_{n}\right)} \sum_{k=1}^{n} e^{2 u_{n, k}} .
$$

Two latter estimates and condition (5) yield

$$
U\left(x_{n}\right) \prec e^{2 \widetilde{u}\left(x_{n}\right)} .
$$

By estimate (9) we arrive at the statement of the theorem. The proof is complete.

Corollary 2. Let $H$ be a radial functional Hilbert space stable with respect to dividing, in which the system of monomials is complete. If condition (7) holds, then the norm of the space $H$ is equivalent to the norm

$$
\|F\|_{0}^{2}:=\frac{1}{2 \pi} \sum_{n=1}^{\infty} e^{-2 \widetilde{u}\left(\ln R_{n}\right)} \int_{0}^{2 \pi}\left|F\left(R_{n} e^{i \varphi}\right)\right|^{2} d \varphi .
$$

This statement is implied by Theorem 4 and Lemma 3 .

\section{BIBLIOGRAPHY}

1. N. Aronszajn. Theory of reproducing kernels // Trans. Amer. Math. Soc. 68:3, 337-404 (1950).

2. S.V. Hruščev, N.K. Nikol'skii, B.S. Pavlov. Unconditional Bases of exponentials and of reproducing kernels // Compl. Anal. Spect. Theor. Lecture Notes in Mathematics. 864, 214-335 (1981).

3. A.F. Leontiev. Exponential series. Nauka, Moscow (1976). (in Russian).

4. Yu.F. Korobeinik Representing systems // Uspekhi Matem. Nauk. 36:1(217), 73-126 (1981). [Russ. Math. Surv. 36:1, 75-137 (1981).]

5. K.P. Isaev. Representing exponential systems in spaces of analytical functions // Itogi Nauki i Tekhniki. Ser. Sovrem. Mat. Pril. Temat. Obz. 161, 3-64 (2019). (in Russian).

6. K.P. Isaev, K.V. Trounov, R.S. Yulmukhametov. Representing systems of exponentials in projective limits of weighted subspaces of $H(D)$ // Izv. RAN. Ser. Matem. 83:2, 40-60 (2019). [Izv. Math. 83:2, 232-250 (2019).] 
7. D.L. Russell. On exponential bases for the Sobolev spaces over an interval // J. Math. Anal. Appl. 87:2, 528-550 (1982).

8. B.Ya. Levin, Yu.I. Lyubarskii. Interpolation by means of special classes of entire functions and related expansions in series of exponentials // Izv. AN SSSR. Ser. Matem. 39:3, 657-702 (1975). [Math. USSR-Izv. 9:3, 621-662 (1975).]

9. K.P. Isaev. Riesz bases of exponents in Bergman spaces on convex polygons // Ufimskij Matem. Zhurn. 2:1, 71-86 (2010). (in Russian).

10. V.I. Lutsenko. Unconditional bases of exponentials in Smirnov spaces. PhD thesis, Inst. Math. USC RAS. (1992). (in Russian).

11. K.P. Isaev, R.S. Yulmukhametov The absence of unconditional bases of exponentials in Bergman spaces on non-polygonal domains // Izv. RAN. Ser. Matem. 71:6, 69-90 (2007). [Izv. Math. 71:6, 1145-1166 (2007).]

12. R.A. Bashmakov, A.A. Makhota, K.V. Trounov On absence conditions of unconditional bases of exponents // Ufimskij Matem. Zhurn. 7:2, 19-34 (2015). [Ufa Math. J. 7:2, 17-32 (2015).]

13. K.P. Isaev. On unconditional exponential bases in weighted spaces on interval of real axis // Lobachevskii Journal of Mathematics. 38:1, 48-61 (2017).

14. K. Seip. Density theorems for sampling and interpolation in the Bargmann-Fock space I // Reine Angew. Math. 429:1, 91-106 (1992).

15. K. Seip, R. Wallsten. Density theorems for sampling and interpolation in the Bargmann-Fock space II // Reine Angew. Math. 429:1, 107-113 (1992).

16. A. Borichev, R. Dhuez, K. Kellay. Sampling and interpolation in large Bergman and Fock spaces // J. Funct. Anal. 242:2, 563-606 (2007).

17. A. Borichev, Yu. Lyubarskii Riesz bases of reproducing kernels in Fock type spaces // Journal of the Institute of Mathematics of Jussieu. 9, 449-461 (2010).

18. A. Baranov, Yu. Belov, A. Borichev. Fock type spaces with Riesz bases of reproducing kernels and de Branges spaces // Studia Mathematica. 236:2, 127-142 (2017).

19. K.P. Isaev, R.S. Yulmukhametov. Unconditional bases in radial Hilbert spaces // Vladikavkaz Matem. Zhurn. 22:3, 85-99 (2020). (in Russian).

20. V.I. Lutsenko, R.S. Yulmukhametov. Generalization of the Paley-Wiener theorem in weighted spaces // Matem. Zametki. 48:5, 80-87 (1990).[Math. Notes. 48:5, 1131-1136 (1990).]

21. R.A. Bashmakov, K.P. Isaev, R.S. Yulmukhametov. On geometric characteristics of convex functions and Laplace integrals // Ufimskij Matem. Zhurn. 2:1, 3-16 (2010). (in Russian).

Konstantin Petrovich Isaev,

Institute of Mathematics,

Ufa Federal Research Center, RAS,

Chernyshevsky str. 112,

450008, Ufa, Russia

Bashkir State University,

Zaki Validi str. 32,

450000, Ufa, Russia

E-mail: orbit81@list.ru

Rinad Salavatovich Yulmukhametov,

Institute of Mathematics,

Ufa Federal Research Center, RAS,

Chernyshevsky str. 112,

450008, Ufa, Russia

E-mail: Yulmukhametov@mail.ru 